\title{
LA GESTIÓN ADMINISTRATIVA \\ DE LA BIBLIOTECA EN UNA \\ UNIVERSIDAD A DISTANCIA
}

\author{
LUZ ADRIANA MARTÍNEZ VARGAS \\ Universidad Estatal a Distancia, Costa Rica \\ Imartinez@uned.ac.cr
}

\section{RESUMEN}

Siendo la biblioteca un recurso indispensable que apoya el desarrollo académico de los estudiantes, es indispensable analizar la gestión administrativa de esta. Con el fin de conocery examinar dicha actividad de las bibliotecas se realizó una investigación que busca analizar la eficacia de los servicios y recursos ofrecidos por la biblioteca de una universidad a distancia (UNED de Costa Rica). Se obtuvo información objetiva tanto de carácter cuantitativo como cualitativo tomando como fuentes las diferentes poblaciones usuarias y las personas funcionarias de esta dependencia. Se utilizaron como técnicas, encuestas, entrevistas, talleres y observaciones obtenidas por las visitas realizadas a las bibliotecas. Los principales resultados recalcan la importancia de una adecuada gestión administrativa para el logro de sus objetivos la cual se concentra en aspectos relacionados con la comunicación, divulgación, cantidad, calidad y actualización del acervo bibliográfico, el personal competente y la necesidad de utilizar herramientas tecnológicas para el desarrollo de la biblioteca tradicional hacia una biblioteca digital. Al analizar la información recabada se destaca la necesidad de elaborar e implementar un plan de mejora que ayude a mejorar la calidad de los servicios bibliotecarios para que estos cumplan con la eficacia requerida.

PALABRAS CLAVE: GESTIÓN ADMINISTRATIVA, SERVICIOS BIBLIOTECARIOS, MEJORA DE LA CALIDAD, RECURSOS BIBLIOGRÁFICOS, USO DE BIBLIOTECA.

\begin{abstract}
Since libraries support the academic development of students, it is essential to analyze all aspects of their administrative management. In order to understand and analyze library management, research was conducted to evaluate the effectiveness of the services and resources offered by the library of the Costa Rican University of Distance Education (UNED, Costa Rica). Enough information was obtained from both quantitative and qualitative sources including the library personnel and by analyzing different user populations. Surveys, interviews, workshops and fields observations were used. The main results emphasize the importance of a proper administrative management for the achievement of the library's objectives. Aspects related to communication, disclosure, quantity, quality and the update of the bibliographical material, staff competence and the technology to development virtual libraries were highlighted. The need to develop and implement an improvement plan to assure the quality of the library services was identified.
\end{abstract}

KEYWORDS: ADMINISTRATIVE MANAGEMENT, LIBRARY SERVICES, QUALITY IMPROVEMENT, LIBRARY RESOURCES, USE OF LIBRARY.

\section{INTRODUCCIÓN}

La biblioteca es un servicio indispensable para las instituciones de educación superior ya que reúne personas, recursos, colecciones tanto físicas como virtuales e infraestructura, cuyo propósito es apoyar a los estudiantes en el proceso de enseñanza y aprendizaje ayudándoles a transformar la información en conocimiento. 
En un sistema de enseñanza a distancia la biblioteca es parte integrante, junto con los materiales, las tutorías y las orientaciones de curso.

La biblioteca debe considerarse como un medio de apoyo por el cual el estudiante puede concretar y ampliar sus conocimientos; esta es parte integral del sistema de aprendizaje haciendo mas efectivo este proceso.

En la enseñanza a distancia el estudiante debe aprender a ser dueño de su propia enseñanza. Por lo tanto, es necesario que adquiera buenos hábitos de lectura y aproveche los recursos didácticos ofrecidos por la universidad, entre los cuales se encuentra la colección de libros e información, tanto física como digital contenida en la biblioteca (Martínez y Chavarría, 2010).

Los estudiantes y los tutores, quienes se encargan de su orientación, deben conocer los recursos educativos disponibles y aprovecharlos al máximo. Ya que en la educación a distancia no se dispone de la presencia de un profesor para resolver las dudas inmediatas, estas solo pueden ser resueltas por los medios con los que cuenta la universidad, entre los que se encuentran los libros de consulta ubicados en las diferentes bibliotecas localizadas en todas las regiones del país.

Debido al rápido desarrollo actual de las ciencias, miles de artículos e información que va apareciendo en revistas y libros se convierten en obsoletos rápidamente; por ende, los tutores, como guía y orientadores del estudiante, deben estar al día sobre lo que acontece en su campo específico para sugerir nuevas lecturas que complementen la información al estudiante que sigue el material didáctico.

Para cumplir este papel deben conocer todas las posibilidades que la biblioteca ofrece, el fondo bibliográfico disponible y participar en la propuesta de las nuevas adquisiciones que se van efectuando. Por su parte, la biblioteca debe ofrecer una gestión administrativa que responda a las necesidades de sus poblaciones meta.
Una de las ventajas de la educación a distancia (Cabral, 2008) es la optimización de los recursos. Lo anterior se logra por medio de la tecnología la cual permite a los estudiantes localizar en un solo sitio la información actualizada que requieren para complementar su formación e incentivar la investigación, lo cual logran en las bibliotecas digitales.

Por su parte, la biblioteca debe ofrecer una amplia labor de divulgación de los materiales que posee, mantener gran flexibilidad en los préstamos de materiales de consulta e incluir dentro de la colección la bibliografía de los cursos que se imparten según la oferta académica de la UNED, como también debe contar con personal capacitado para orientar a los usuarios en la obtención de la información tanto física como digital.

Teniendo en cuenta los temas mencionados anteriormente se realizó una investigación para conocer cómo se encuentran las bibliotecas de una Universidad a distancia (UNED de Costa Rica) en cuanto a su gestión administrativa y si está realmente cubriendo las necesidades de las poblaciones usuarias.

El principal objetivo ha lograr fue analizar la eficacia de la gestión administrativa de la biblioteca para ofrecer los servicios a sus poblaciones meta, esto teniendo en cuenta los recursos con los que cuenta y sus procesos. Para lograr este objetivo se analizó la valoración del personal colaborador de la biblioteca y de las personas usuarias en cuanto a la disponibilidad, accesibilidad y suficiencia de los servicios, capacidad instalada y recursos bibliográficos.

Para lograr los objetivos propuestos se establecieron las siguientes variables (Martínez y Chavarría, 2010):

- Uso: especificando la frecuencia del mismo y los medios de uso (presencial o remoto).

- Infraestructura: en cuanto a las instalaciones se incluyeron los aspectos relacionados con ambiente, orden, comodidad, número de puestos 
para estudio, horario y señalización. En cuanto a computadoras, aspectos como número de puestos, estado de las computadoras, actualización de las mismas y acceso a internet.

- Recursos bibliográficos: actualización, estado y cantidad de los mismos y políticas de actualización.

- Facilidades para las personas usuarias: servicio, préstamo y formación e información para los usuarios.

- Sistema de información: instrucciones, actualidad, agilidad, facilidad de uso.

- Divulgación: estrategias de divulgación.

\section{METODOLOGÍA}

Para realizar esta investigación se recolectó y sistematizó información objetiva de carácter cuantitativo y cualitativo que incluyó información sobre la realidad de las bibliotecas de la UNED integrada por datos e información sobre los procesos y resultados de la biblioteca, observaciones, opiniones y valoraciones (Martínez y Chavarría, 2010).
Para la recolección de la información necesaria se utilizó la revisión documental, adicionalmente se adaptó a las características de la UNED la Guía para la evaluación de bibliotecas españolas elaborada por la Agencia para la calidad del Sistema Universitario de Cataluña (2002).

Esta guía incluye aspectos relacionados con la biblioteca y su integración con el marco institucional, los procesos y la comunicación, los recursos de la misma y los resultados centrados en la satisfacción de los usuarios.

Para complementar la información requerida por la guía se utilizó documentación oficial y operativa de la biblioteca, datos de los servicios ofrecidos, observaciones sobre la realidad de la biblioteca y opiniones y valoraciones de las poblaciones usuarias las cuales fueron recopiladas por medio de entrevistas, talleres grupales y encuestas.

\section{Población de estudio e instrumentos aplicados}

En la Tabla 1 se detallan las poblaciones incluidas en el estudio y el instrumento aplicado a cada una de ellas.

TABLA 1

\section{POBLACIÓN E INSTRUMENTOS}

\begin{tabular}{|l|l|l|}
\hline \multicolumn{1}{|c|}{ Población } & \multicolumn{1}{|c|}{ Muestra } & \multicolumn{1}{c|}{ Instrumento } \\
\hline $\begin{array}{l}\text { Estudiantes matriculados } \\
\text { del segundo cuatrimestre } \\
\text { del 2008 al al primer } \\
\text { cuatrimestre del } 2009\end{array}$ & $\begin{array}{l}114 \text { estudiantes de matrícula ordinaria y 62 de extensión, } \\
\text { teniendo en cuenta un ajuste de no respuesta del 30\%. } \\
\text { Estos pertenecen a diferentes lugares del país y cursan varia- } \\
\text { das carreras. }\end{array}$ & $\begin{array}{l}\text { Encuesta } \\
\text { telefónica }\end{array}$ \\
\hline $\begin{array}{l}\text { Investigadores } \\
44 \text { personas }\end{array}$ & Encuesta \\
\hline $\begin{array}{l}\text { Funcionarios que usan la } \\
\text { biblioteca en la sede central }\end{array}$ & 29 personas & Encuesta \\
\hline Tutores & 56 tutores & Encuesta \\
\hline $\begin{array}{l}\text { Encargados de cátedra } \\
\text { y programa }\end{array}$ & 23 personas & Dos talleres \\
\hline Comunidad & 33 personas de diferentes comunidades & Encuesta \\
\hline Funcionarios de la biblioteca & 28 funcionarios & Encuesta \\
\hline Jefatura de la biblioteca & 1 persona & $\begin{array}{l}\text { Entrevista } \\
\text { observaciones }\end{array}$ \\
\hline Centros universitarios & 17 centros universitarios & \\
\hline
\end{tabular}

Fuente: Martínez y Chavarría (2010). 
Es importante recalcar que los miembros de la comunidad que se tuvieron en cuenta en este estudio son personas que utilizan las bibliotecas de los centros universitarios.

\section{La Universidad Estatal a Distancia de Costa Rica}

La UNED fue creada en el año 1977 y es una universidad pública con un modelo pedagógico basado exclusivamente en la enseñanza a distancia, para lo cual hace uso de los diversos medios tecnológicos y de comunicación social. Debido a su modalidad y como apoyo para el autoaprendizaje la universidad da a sus estudiantes el material requerido para su aprendizaje el cual es denominado unidades didácticas modulares. Cuenta con 37 Centros Universitarios distribuidos en todo el país en los cuales se ofrecen los diferentes servicios a los estudiantes y a la comunidad, en el caso de los estudiantes en estas sedes se ofrecen tutorías presenciales, las cuales son guiadas por tutores, estos tutores pertenecen a una cátedra específica y son dirigidos por un Encargado de Cátedra, una cátedra está conformada por un grupo de cursos. Cada uno de estos centros cuenta con un administrador que se encarga de la gestión del mismo. La universidad ofrece sus carreras por medio de cuatrimestres y entre ellas se encuentran carreras o programas de pregrado y de posgrado, también ofrece cursos de extensión para todas las regiones del país.

\section{La biblioteca de la UNED}

En 1978 surge la Biblioteca Central de la UNED así como la Red de Bibliotecas de los Centros universitarios, que pretendía beneficiar a los estudiantes, funcionarios y comunidad en general. Los servicios de la misma fueron creciendo al incrementar el número de centros universitarios. Posteriormente la biblioteca fue incursionando en la tecnología al adquirir un sistema de infor- mación especializado que apoyara las tareas de esta entidad (Martínez y Chavarría, 2010).

Actualmente, el Sistema de Bibliotecas de la UNED está compuesto por una Biblioteca Central y bibliotecas en 30 centros universitarios.

La Biblioteca Central ubicada en la sede central es la encarga de los procesos de selección, adquisición, catalogación, clasificación y distribución del material que utilizará todas las bibliotecas del sistema. Aquí también establecen las directrices de trabajo de todas la bibliotecas ubicadas en todas las regiones del país, se dictan las políticas en lo referente a servicios y capacitación del personal (Martínez y Chavarría, 2010).

De forma general, los servicios que presta la biblioteca se centran en: préstamo en sala, préstamo a domicilio, préstamo entre el sistema bibliotecario de la UNED, préstamo interbibliotecario, copia de videos, recursos electrónicos, catálogo en línea y biblioteca digital. Estos servicios la biblioteca se los ofrece a todas las poblaciones de la Universidad: a estudiantes, funcionarios y a particulares que los requieran.

Las bibliotecas localizadas en los diferentes centros universitarios ofrecen todos servicios ofrecidos en su sede central, solo que en la mayoría de estos centros universitarios no se cuenta con personal especializado en bibliotecología, por lo que las mismas son atendidas por personal polifuncional, y en algunos casos no se cuenta con personal a tiempo completo. Uno de los mayores inconvenientes es que el personal que trabaja en estas bibliotecas tiene como jefe a los administradores de centros y solamente tienen la dirección técnica por parte de la jefatura de la biblioteca (Martínez y Chavarría, 2010).

La provisión de materiales de las diferentes bibliotecas es responsabilidad de la biblioteca central, ya que esta es la responsable de la compra de los materiales teniendo como base las solicitudes de las Escuelas. 


\section{Las bibliotecas universitarias}

Las bibliotecas, especialmente las universitarias, cumplen unos objetivos específicos como lo detalla González y Molina (2008):

Las bibliotecas universitarias son instituciones que reúnen, organizan y difunden información para el aprendizaje, la docencia y la investigación, y potencian la creación de nuevos conocimientos..., las bibliotecas universitarias también puede verse como centros que contribuyen al desarrollo de la cultura y la transmisión de los valores locales hacia el entorno de la comunidad donde se encuentran situadas, y esto genera un fuerte impacto no sólo cultural sino también social (p. 3-4).

Desde esta visión, la biblioteca universitaria debe procurar apoyar a las instituciones a las cuales pertenece, así como también proyectarse y prestar sus servicios a la comunidad a la cual pertenecen con el fin de ayudar en el desarrollo de las mismas.

Para el logro de sus objetivos estas entidades deben contar con el personal adecuado como indica Montagut (2009): "el bibliotecario también es clave fundamental, de ahí que su capacitación sea importante" (p. 55).

\section{La gestión administrativa de las bibliotecas}

Una biblioteca es un ente que bajo cierta dirección y administración busca satisfacer las necesidades de información de sus poblaciones meta, para lograr sus objetivos requiere establecer e implementar herramientas que le permitan administrar sus procesos de manera que estos se desarrollen de la mejor manera. Por lo tanto, requiere de instalaciones adecuadas, personal competente, fondos bibliográficos que respondan a las necesidades de sus poblaciones, recur- sos económicos y tecnológicos(los cuales están influenciando en gran medida los cambios de las nuevas bibliotecas). Adicionalmente requieren el establecimiento y administración de procesos que permitan el logro de sus objetivos. Los procesos de una biblioteca se pueden clasificar en 3 áreas: área administrativa, procesamiento de los documentos y servicio al público. En las tres áreas el apoyo informático es indispensable para su desarrollo y para garantizar un buen servicio al usuario (Gutiérrez y Zárate, 2007).

Lo anterior confirma la necesidad que dentro de una biblioteca se requiere de una adecuada gestión de estos elementos para prestar sus servicios. Esta gestión debe incluir aspectos de planificación, organización, dirección y control.

\section{La gestión de la calidad en bibliotecas}

Desde la década de los 90 (González y Molina, 2008) se introdujo en el funcionamiento de las bibliotecas conceptos de la administración como los relacionados con planificación estratégica, planificación por objetivos y las técnicas de gestión de calidad, buscando la satisfacción de los usuarios.

Dentro de los conceptos de gestión de calidad, indican Gonzales y Molina (2008), que implica la mejora en todos los aspectos de gestión, procesos, servicios, infraestructura y planificación entre otros, también se destaca el concepto de evaluación de la calidad por medio del cual se busca dar a conocer que la biblioteca trabaja de forma trasparente y logra la satisfacción de sus usuarios como la mejora constante de la misma ubicando puntos débiles y trabajando sobre los mismos y resaltando las buenas acciones que ayudan a su funcionamiento.

Actualmente, la evaluación de una biblioteca debe basarse en información tanto cuantitativa como cualitativa de la satisfacción de los usuarios, teniendo siempre presente el entorno bajo 
el cual funciona e involucrando a todo su personal ya que de ellos depende la ejecución de las mejoras requeridas. Una herramienta básica y de gran utilidad para evaluar bibliotecas es la utilización de indicadores tanto de servicio como internamente de personal.

\section{Las bibliotecas actuales}

Las bibliotecas de esta generación según González y Molina (2008) deben:

Automatizar sus procesos internos, crear áreas de autoaprendizaje y puestos de acceso a internet en sus salas de lectura, facilitar el acceso de los profesores e investigadores a la producción científica, crear repositorios de materiales docentes, ofrecer servicios en línea de préstamo, información y referencia y videoteca digital, entre otros (p. 4).

Debido a los cambios actuales en la sociedad y en la generación de la llamada "sociedad de la información y el conocimiento", en la biblioteca, según Orera (2007), se debe trabajar en la gestión de la información digital generando un nuevo modelo denominado "biblioteca híbrida" en la que se combinan tanto elementos tradicionales como nuevos relacionados directamente con la información digital y las nuevas tecnologías, que le permitan a estas adaptarse a los cambios requeridos para poder seguir existiendo y apoyando la generación de conocimiento y el aprendizaje en las universidades, de manera que se atiendan usuarios tanto presenciales como remotos. Para evitar la desaparición de la biblioteca tradicional y para ofrecer servicios de acuerdo a las condiciones actuales es necesario la continua actualización de las mismas sin olvidar su esencia como administradores de la información.

La biblioteca digital comenzó a surgir desde la década de 1990 y tiene como fin ofrecer servicios acordes con las demandas actuales de los usuarios los cuales solicitan colecciones digitales y servicios complementarios, entre los que se encuentran revistas electrónicas y directorios temáticos especializados (Tramullas, 2007). Una biblioteca digital permite el acceso a la información por medio de internet debido a que los documentos se encuentran en formatos digitales y no físicos pero de igual manera cuentan con alguna sede geográfica, lo que la diferencia de una biblioteca virtual la cual no existe físicamente, como lo señala Sánchez y Vega (2002) la biblioteca virtual "nunca tuvo colección impresa ni edificio y se crea a partir de documentos digitalizados y sitios" (p. 7).

Como lo indican Sánchez y Vega (2002), en la página web de una biblioteca digital no es necesario que se encuentren todos los textos que esta ofrece, puede ofrecer solamente las listas de enlaces con ciertas clasificaciones por temas.

Un punto primordial, señala Orera (2007), de la nueva generación de bibliotecas es el personal que estas requieren para su funcionamiento ya que la selección de la información y su gestión requiere de personal muy calificado y con conocimientos en informática y en capacitación constante. El personal que labora en estas entidades se ve en la obligación de cambiar su perfil para adaptarse a las necesidades actuales del entorno.

Uno de los elementos para lograr calidad en las bibliotecas de la actualidad (González \& Molina, 2008) es contar con profesionales que adicional a las capacidades de un bibliotecólogo: dominen el contenido de las fuentes de información, tengan capacidad para evaluarlos y filtrarlos, utilicen la tecnología, diseñen servicios, planteen estrategias de divulgación, manejen lo relacionado con la propiedad intelectual y realicen investigaciones; de manera que sea un asesor de la información que apoye a los docentes y estudiantes en la generación de nuevos conocimientos 
Como indica Orera (2007), las nuevas bibliotecas ya no se basan en tener su propia colección para ofrecerla a sus usuarios sino facilitar el acceso a colecciones digitales. Debido a esto, se requiere un cambio en cuanto a infraestructura y equipo de manera que puedan adaptarse a estos cambios y cumplir con el usuario.

Para la Research Planning and Review Committee (ACRL, 2010), entre las 10 principales tendencias en las bibliotecas universitarias se destacan los cambios presupuestarios que las afectarán, la necesidad de ampliar las competencias y habilidades de los bibliotecarios, el incremento de la solicitud de rendición de cuentas, aumento de la digitalización de las colecciones de libros, el rápido crecimiento del uso de dispositivos móviles y sus aplicaciones, recalcar la importancia de los derechos de autor, cambios tecnológicos, disminución de los espacios físicos para las colecciones y aumento de recursos en línea.

\section{La biblioteca en la educación a distancia}

Como indican Martínez y Chavarría (2010) la biblioteca en el sistema de educación a distancia es una herramienta más para poder obtener información necesaria para la formación de los estudiantes y según Padula (2002):

La educación a distancia, no presencial o de reducida presencialidad debe brindar la mayor cantidad posible de contenidos a través de los materiales cuya provisión sistematiza, ya que presupone distancias espacio-temporales que separan al alumno del entorno pedagógico basado en la relación cara a cara. Sin embargo, el método no puede abarcar todos los contenidos conceptuales a través de materiales de factoría propia (p. 8).

Al analizar el caso de la UNED y su sistema educativo en el cual las unidades didácticas son las que guían el aprendizaje de los estudiantes, es muy probable que no se puedan incluir todos los contenidos en las mismas o que no se pueda actualizar tan constantemente, así como también la necesidad de la consulta de versiones originales y de desarrollar habilidades en el estudiante como lo es la investigación, lo cual requiere material adicional (Martínez y Chavarría, 2010).

Lo que implica que una universidad de este tipo debe tener todos los recursos necesarios para ofrecer servicios bibliotecarios útiles y actualizados de acuerdo a las nuevas tendencias en este aspecto.

Según Cabral (2008) "La educación a distancia, comparada con la tradicional, presenta una serie de ventajas tanto para los educandos como para las instituciones educativas: la flexibilidad de horarios, en el primer caso, y la optimización de recursos, en el segundo" (p. 64).

Dentro de esta optimización de recursos se debe incluir los servicios bibliotecarios que apoyan el aprendizaje y en la actualidad se materializa en la biblioteca digital y como indica Cabral (2008) "La biblioteca digital proporciona los insumos necesarios para que una modalidad a distancia funcione de manera óptima" (p. 67).

Entre otras ventajas de este tipo de biblioteca Cabral (2008) añade "la biblioteca digital permite la interacción entre los usuarios, los docentes y los contenidos, facilitando la colaboración, además de fomentar la investigación y la generación de nuevos conocimientos, por mencionar sólo algunas de sus aportaciones a la educación a distancia" (p. 68).

\section{Uso de las bibliotecas de la UNED y los servicios que ofrece}

Una de las variables a analizar en el estudio se refiere al uso que las diferentes poblaciones hacen de este servicio, el cual se presenta en la Tabla 2. 


\section{USO DE LAS BIBLIOTECAS DE LA UNED Y SUS SERVICIOS}

\begin{tabular}{|c|c|c|c|}
\hline $\begin{array}{l}\text { Poblaciones } \\
\text { consultadas }\end{array}$ & $\begin{array}{l}\text { Frecuencia de uso de las } \\
\text { instalaciones de la biblioteca }\end{array}$ & $\begin{array}{l}\text { Uso del equipo de } \\
\text { cómputo de la biblioteca }\end{array}$ & $\begin{array}{l}\text { Forma de uso } \\
\text { de la biblioteca }\end{array}$ \\
\hline Investigadores & $\begin{array}{l}\text { - } 65 \% \text { una a dos veces al mes } \\
-20 \% \text { nunca } \\
-10 \% \text { una o dos veces por semana } \\
\text { - } 5 \% \text { tres o mas veces por semana }\end{array}$ & $\begin{array}{l}\text { - } 47 \% \text { menos de una vez al mes } \\
-35 \% \text { una vez al mes } \\
-12 \% \text { dos o mas veces por semana } \\
\text { - } 6 \% \text { una vez por semana }\end{array}$ & $\begin{array}{l}\text { - } 73 \% \text { presencial en la } \\
\text { sede central } \\
\text { - } 23 \% \text { virtual } \\
\text { - } 4 \% \text { presencial en el } \\
\text { centro universitario }\end{array}$ \\
\hline Estudiantado & $\begin{array}{l}-91 \% \text { nunca } \\
-4 \% \text { solo en época de tarea } \\
-2 \% \text { una a dos veces al mes } \\
-2 \% \text { tres o mas veces por semana } \\
\text { - } 1 \% \text { una o dos veces por semana }\end{array}$ & $\begin{array}{l}\text { - } 50 \% \text { menos de una vez al mes } \\
-25 \% \text { una vez al mes } \\
-25 \% \text { dos o mas veces por semana }\end{array}$ & $\begin{array}{l}\text { - } 82 \% \text { presencial en } \\
\text { el centro universitario } \\
\text { - } 18 \% \text { virtual }\end{array}$ \\
\hline $\begin{array}{l}\text { Funcionarios } \\
\text { de la Sede Central }\end{array}$ & $\begin{array}{l}-69 \% \text { una a dos veces al mes } \\
-10 \% \text { pocas veces } \\
-7 \% \text { nunca } \\
-7 \% \text { tres o mas veces por semana } \\
-7 \% \text { no responde }\end{array}$ & $\begin{array}{l}-42 \% \text { menos de una vez al mes } \\
-27 \% \text { una vez al mes } \\
-23 \% \text { no responde } \\
-4 \% \text { dos o mas veces por semana } \\
-4 \% \text { una vez por semana }\end{array}$ & $\begin{array}{l}\text { - } 64 \% \text { presencial en } \\
\text { la sede central } \\
\text { - } 36 \% \text { virtual }\end{array}$ \\
\hline Tutores & $\begin{array}{l}-64 \% \text { nunca } \\
\text { - } 30 \% \text { una a dos veces al mes } \\
\text { - } 4 \% \text { una o dos veces por semana } \\
\text { - } 2 \% \text { tres o mas veces por semana }\end{array}$ & $\begin{array}{l}-60 \% \text { menos de una vez al mes } \\
-25 \% \text { una vez al mes } \\
-10 \% \text { no responde } \\
-5 \% \text { dos o mas veces por semana }\end{array}$ & $\begin{array}{l}-39 \% \text { presencial en } \\
\text { la sede central } \\
\text { - } 32 \% \text { virtual } \\
\text { - } 29 \% \text { presencial en } \\
\text { el centro universitario }\end{array}$ \\
\hline Comunidad & $\begin{array}{l}\text { - } 67 \% \text { una a dos veces al mes } \\
-15 \% \text { una o dos veces por semana } \\
\text { - } 9 \% \text { tres o mas veces por semana } \\
-9 \% \text { no responde }\end{array}$ & No aplica & $\begin{array}{l}\text { - } 87 \% \text { presencial en } \\
\text { el centro universitario } \\
-13 \% \text { virtual }\end{array}$ \\
\hline
\end{tabular}

Fuente: Martínez y Chavarría (2010).

Para complementar la información suministrada en la Tabla 2 se indica que entre las razones de no uso de la biblioteca en la población estudiantil un aspecto a destacar es que no la necesitan, les es suficiente con la unidad didáctica y utilizan otros medios para acceder a los libros (Martínez y Chavarría, 2010).

Al analizar la información obtenida se observa que las poblaciones consultadas hacen poco uso de los servicios y equipos de la biblioteca.
Adicionalmente es concluyente que las poblaciones que hacen uso de las bibliotecas lo hacen con muy poca frecuencia y la mayoría de manera presencial indicando esto el poco conocimiento de los servicios virtuales que ofrecen las bibliotecas y de cómo utilizarlos.

En cuanto a la variable relacionada con la formación e información para los usuarios de las bibliotecas de la UNED se obtuvieron los resultados presentados en la Tabla 3. 


\section{TABLA 3 \\ RESULTADOS VALORACIÓN USUARIOS BIBLIOTECAS DE LA UNED SOBRE LA INFORMACIÓN Y FORMACIÓN DE USUARIOS}

\begin{tabular}{|c|c|}
\hline $\begin{array}{l}\text { Poblaciones } \\
\text { consultadas }\end{array}$ & Formación e información para los usuarios de la biblioteca \\
\hline Investigadores & $\begin{array}{l}\text { - 81\% opina que no se les ha brindado información acerca de los servicios que } \\
\text { ofrece la biblioteca. } \\
\text { - } 19 \% \text { opinan que si se les brindó información. }\end{array}$ \\
\hline Estudiantado & $\begin{array}{l}\text { - } 62 \% \text { opina que no se les informo acerca de los servicios que ofrece la biblioteca. } \\
\text { - } 38 \% \text { opinan que si se les brindó información. }\end{array}$ \\
\hline $\begin{array}{l}\text { Funcionarios } \\
\text { de la Sede Central }\end{array}$ & $\begin{array}{l}\text { - 77\% opina que no recibió información sobre la biblioteca cuando ingreso a la UNED. } \\
\text { - 23\% opinan que si se les brindó información. }\end{array}$ \\
\hline Tutores & $\begin{array}{l}\text { - 80\% opina que no se les ha brindado información acerca de los servicios que } \\
\text { ofrece la biblioteca. } \\
\text {-20 opinan que si se les brindó información. }\end{array}$ \\
\hline
\end{tabular}

Fuente: Martínez y Chavarría (2010).

Los resultados de la Tabla 3 demuestran que los esfuerzos que ha realizado la biblioteca para formar e informar a los usuarios sobre cómo acceder a la misma, ya sea física o virtualmente y sobre los servicios que ofrece, no han suficientes, ya que no están llegando a los usuarios.

Por otro lado el sistema de información con que cuentan las bibliotecas abarca la base de datos de ésta, la cual incluye el inventario de todos los libros e información física que contiene y que es utilizado para ubicar los mismos tanto por los funcionarios de la biblioteca como por los usuarios.

Al consultar a los colaboradores de las bibliotecas sobre la utilidad y actualidad del sistema de información, se obtuvo que el $61 \%$ de las personas opinan que éste es adecuado (Martínez y Chavarría, 2010). En cuanto a la valoración realizada por las poblaciones usuarias del mismo se presentan los resultados en la Tabla 4. 
TABLA 4

\section{RESULTADOS VALORACIÓN USUARIOS DE LAS BIBLIOTECAS DE LA UNED SOBRE EL SISTEMA DE INFORMACIÓN DE LAS BIBLIOTECAS}

\begin{tabular}{|c|c|c|}
\hline $\begin{array}{l}\text { Poblaciones } \\
\text { consultadas }\end{array}$ & $\begin{array}{l}\text { Instrucciones, actualización } \\
\text { y agilidad del sistema de información }\end{array}$ & $\begin{array}{l}\text { Facilidad para consultar la } \\
\text { base de datos por Internet }\end{array}$ \\
\hline Investigadores & $\begin{array}{l}-71 \% \text { positivo } \\
-10 \% \text { negativo } \\
-19 \% \text { no responde }\end{array}$ & $\begin{array}{l}-55 \% \text { regularmente satisfechos } \\
-30 \text { satisfechos } \\
-15 \% \text { insatisfechos }\end{array}$ \\
\hline Estudiantado & $\begin{array}{l}-70 \% \text { no responde } \\
-26 \% \text { negativo } \\
-4 \% \text { positivo }\end{array}$ & $\begin{array}{l}\text { - } 40 \% \text { Satisfechos } \\
\text { - } 30 \% \text { regularmente satisfechos } \\
\text { - } 30 \% \text { insatisfechos }\end{array}$ \\
\hline $\begin{array}{l}\text { Funcionarios } \\
\text { de la Sede Central }\end{array}$ & $\begin{array}{l}-53 \% \text { positivo } \\
-30 \% \text { no responde } \\
-17 \% \text { negativo }\end{array}$ & $\begin{array}{l}\text { - } 59 \% \text { regularmente satisfechos } \\
\text { - } 45 \% \text { satisfechos } \\
-6 \% \text { insatisfechos }\end{array}$ \\
\hline Tutores & $\begin{array}{l}-56 \% \text { positivo } \\
-32 \% \text { no responde } \\
-12 \% \text { negativo }\end{array}$ & $\begin{array}{l}\text { - } 54 \% \text { regularmente satisfechos } \\
\text { - } 40 \% \text { satisfechos } \\
-6 \% \text { insatisfechos }\end{array}$ \\
\hline Comunidad & No aplica & $\begin{array}{l}-67 \% \text { satisfechos } \\
-15 \% \text { insatisfechos } \\
-18 \% \text { no responde }\end{array}$ \\
\hline
\end{tabular}

Fuente: Martínez y Chavarría (2010).

\section{Los recursos de la biblioteca de la UNED}

Como indican Martínez y Chavarría (2010):

...en cuanto al personal de la biblioteca es importante recalcar el hecho que las personas encargadas de las bibliotecas en los centro universitarios no tienen la formación requerida para realizar sus funciones de una manera eficaz, aspecto que lo detectan los encargados de cátedra y de programa, adicionalmente estas personas colaboradoras indican la necesidad de recibir capacitación y seguimiento relacionados a su quehacer. Aunque la jefatura de la biblioteca organiza ciertas capacitaciones, no están incluidas en un plan de capacitación que ayude de manera sistemática a la formación de sus colaboradores. Lo que le ha ayudado a la mayoría de las personas funcionarias es la experiencia ya que llevan varios años laborando en este lugar (p. 42).
Como parte de la investigación se realizaron visitas a las bibliotecas de algunos centros universitarios, en los cuales se observó que en la mayoría los colaboradores no cuentan con el espacio adecuado para realizar sus funciones. En relación con el espacio para la consulta de los usuarios, la mayoría de las bibliotecas visitadas no tienen un espacio adecuado y casi todas no cuentan con aire acondicionado, salas para consultas y estudio. En lo que respecta a las condiciones ambientales, 66\% indica que estas son algo y poco adecuadas para realizar sus funciones (Martínez y Chavarría, 2010).

Los resultados obtenidos a partir de la información dada por las poblaciones usuarias en cuanto a las variables de instalaciones y equipo de cómputo ubicado en las bibliotecas se muestran en la Tabla 5. 
TABLA 5

\section{RESULTADOS VALORACIÓN USUARIOS DE LAS BIBLIOTECAS DE LA UNED SOBRE LAS INSTALACIONES Y EL EQUIPO DE CÓMPUTO}

\begin{tabular}{|c|c|c|c|}
\hline $\begin{array}{l}\text { Poblaciones } \\
\text { consultadas }\end{array}$ & $\begin{array}{l}\text { Instalaciones } \\
\text { de la biblioteca }\end{array}$ & $\begin{array}{l}\text { Equipo informático } \\
\text { de la biblioteca }\end{array}$ & $\begin{array}{l}\text { Acceso a internet } \\
\text { en la biblioteca }\end{array}$ \\
\hline Investigadores & $\begin{array}{l}\text { - } 52 \% \text { regularmente } \\
\text { satisfechos } \\
\text { - } 24 \% \text { insatisfechos } \\
\text { - } 24 \% \text { satisfechos }\end{array}$ & $\begin{array}{l}\text { - } 60 \% \text { regularmente } \\
\text { satisfechos } \\
\text { - } 31 \% \text { insatisfechos } \\
\text { - } 9 \% \text { satisfechos }\end{array}$ & $\begin{array}{l}-52 \% \text { regularmente satisfechos } \\
-32 \% \text { insatisfechos } \\
-16 \% \text { satisfechos }\end{array}$ \\
\hline Estudiantado & $\begin{array}{l}\text { - } 50 \% \text { satisfechos } \\
\text { - } 24 \% \text { regularmente } \\
\text { satisfechos } \\
\text { - } 26 \% \text { insatisfechos }\end{array}$ & $\begin{array}{l}\text { - } 56 \% \text { regularmente } \\
\text { satisfechos } \\
\text { - } 35 \% \text { satisfechos } \\
-9 \% \text { insatisfechos }\end{array}$ & $\begin{array}{l}\text { - } 46 \% \text { satisfechos } \\
\text { - } 36 \% \text { regularmente satisfechos } \\
\text { - } 18 \% \text { insatisfechos }\end{array}$ \\
\hline $\begin{array}{l}\text { Funcionarios } \\
\text { de la Sede Central }\end{array}$ & $\begin{array}{l}\text { - } 56 \% \text { satisfechos } \\
\text { - } 36 \% \text { regularmente } \\
\text { satisfechos } \\
\text { - } 8 \% \text { ilnsatisfechos }\end{array}$ & $\begin{array}{l}\text { - } 45 \% \text { regularmente } \\
\text { satisfechos } \\
-33 \% \text { insatisfechos } \\
-22 \% \text { satisfechos }\end{array}$ & $\begin{array}{l}\text { - } 43 \% \text { satisfechos } \\
\text { - } 33 \% \text { insatisfechos } \\
\text { - } 24 \% \text { regularmente satisfechos }\end{array}$ \\
\hline Tutores & $\begin{array}{l}\text { - } 47 \% \text { satisfechos } \\
\text { - } 41 \% \text { regularmente } \\
\text { satisfechos } \\
\text { - } 12 \% \text { insatisfechos }\end{array}$ & $\begin{array}{l}\text { - } 57 \% \text { regularmente } \\
\text { satisfechos } \\
-22 \% \text { satisfechos } \\
-21 \% \text { insatisfechos }\end{array}$ & $\begin{array}{l}\text { - } 52 \% \text { regularmente satisfechos } \\
-27 \% \text { satisfechos } \\
-21 \% \text { insatisfechos }\end{array}$ \\
\hline Comunidad & $\begin{array}{l}\text { - } 69 \% \text { satisfechos } \\
\text { - } 23 \% \text { insatisfechos } \\
\text { - } 8 \% \text { no responde }\end{array}$ & $\begin{array}{l}-64 \% \text { satisfechos } \\
-23 \% \text { insatisfechos } \\
-13 \% \text { no responde }\end{array}$ & $\begin{array}{l}\text { - } 51 \% \text { satisfechos } \\
\text { - 26\% insatisfechos } \\
\text { - } 23 \% \text { no responde }\end{array}$ \\
\hline
\end{tabular}

Fuente: Martínez y Chavarría (2010).

Como lo indica la información de la Tabla 5, las instalaciones no cumplen eficazmente con las necesidades de los usuarios ni de los colaboradores debido a que las mismas son muy pequeñas y no cuentan con las condiciones necesarias para el adecuado almacenamiento de los libros.

En cuanto al equipo informático, se obtuvo que las bibliotecas cuentan con equipo informático adecuado para las labores de los colaboradores, pero el equipo requerido para el uso de los usuarios no es el adecuado y no satisface las necesidades de los mismos.
Uno de los servicios básicos actualmente en las bibliotecas son equipos adecuados y buen acceso a internet, ya que por estos dos medios se puede accesar a la biblioteca digital y la investigación demuestra que los usuarios de las bibliotecas indican que el servicio de acceso a internet requiere ser mejorado.

En cuanto a los fondos bibliográficos, según Martínez y Chavarría (2010) se especifica que el proceso de adquisición como tal es claro, pero no existe una política institucional para la adquisición de los recursos bibliográficos, por lo que este proceso es desvinculado de las escuelas ya 
que se compra de acuerdo a estadísticas y percepción de la biblioteca, por lo que no se logra asegurar la calidad y pertinencia de los materiales bibliográficos, tanto para docencia como para las líneas de investigación de la universidad. Institucionalmente no se cuenta con mecanismos para valorar la calidad y la adecuación de los materiales con la oferta académica de la Universidad y la investigación (p. 45).

Debido a la forma como son distribuidos los materiales a lo largo del país, los mismos no se encuentran distribuidos equitativamente, de manera que no en todos los centros universi- tarios se pueden encontrar los mismos libros y materiales, esto en cuanto al material físico.

Debido a que los encargados de cátedra y programa son la principal fuente de información para alimentar adecuadamente las bibliotecas de acuerdo a la necesidad de los diferentes cursos, el involucramiento de este personal es vital para una adecuada actualización de las bibliotecas y en el estudio se destaca su poca participación en este proceso.

La valoración de los usuarios en cuanto a los fondos bibliográficos se presenta en el Tabla 6 .

TABLA 6

\section{RESULTADOS VALORACIÓN USUARIOS DE LAS BIBLIOTECAS DE LA UNED SOBRE LOS FONDOS BIBLIOGRÁFICOS}

\begin{tabular}{|c|c|c|c|c|}
\hline $\begin{array}{c}\text { Grupos } \\
\text { consultados }\end{array}$ & $\begin{array}{l}\text { Actualización de los } \\
\text { fondos bibliográficos }\end{array}$ & $\begin{array}{l}\text { Estado de los fondos } \\
\text { bibliográficos }\end{array}$ & $\begin{array}{l}\text { Cantidad de los fon- } \\
\text { dos bibliográficos }\end{array}$ & $\begin{array}{c}\text { Políticas de } \\
\text { actualización de los } \\
\text { fondos bibliográficos }\end{array}$ \\
\hline Investigadores & $\begin{array}{l}\text { - } 47 \% \text { insatisfechos } \\
\text { - } 39 \% \text { regularmente } \\
\text { satisfechos } \\
\text { - } 14 \% \text { satisfechos }\end{array}$ & $\begin{array}{l}\text { - } 64 \% \text { regularmente } \\
\text { satisfechos } \\
\text { - } 22 \% \text { satisfechos } \\
\text { - } 14 \% \text { insatisfechos }\end{array}$ & $\begin{array}{l}\text { - } 50 \% \text { insatisfechos } \\
\text { - } 36 \% \text { regularmente } \\
\text { satisfechos } \\
\text { - } 14 \% \text { satisfechos }\end{array}$ & $\begin{array}{l}\text { - } 44 \% \text { regularmente } \\
\text { satisfechos } \\
\text { - } 36 \% \text { satisfechos } \\
-20 \% \text { insatisfechos }\end{array}$ \\
\hline Estudiantado & $\begin{array}{l}\text { - } 73 \% \text { regularmente } \\
\text { satisfechos } \\
\text { - } 18 \% \text { insatisfechos } \\
-9 \% \text { satisfechos }\end{array}$ & $\begin{array}{l}\text { - } 80 \% \text { regularmente } \\
\text { satisfechos } \\
\text { - } 20 \% \text { insatisfechos }\end{array}$ & $\begin{array}{l}\text { - } 50 \% \text { regularmente } \\
\text { satisfechos } \\
\text { - } 33 \% \text { satisfechos } \\
\text { - } 17 \% \text { insatisfechos }\end{array}$ & $\begin{array}{l}\text { - } 60 \% \text { regularmente } \\
\text { satisfechos } \\
\text { - } 40 \% \text { satisfechos }\end{array}$ \\
\hline $\begin{array}{l}\text { Funcionarios } \\
\text { de la Sede } \\
\text { Central }\end{array}$ & $\begin{array}{l}\text { - } 48 \% \text { regularmente } \\
\text { satisfechos } \\
\text { - } 29 \% \text { satisfechos } \\
\text { - } 23 \% \text { insatisfechos }\end{array}$ & $\begin{array}{l}\text { - } 58 \% \text { satisfechos } \\
\text { - } 42 \% \text { regularmente } \\
\text { satisfechos }\end{array}$ & $\begin{array}{l}\text { - } 44 \% \text { regularmente } \\
\text { satisfechos } \\
\text { - } 30 \% \text { satisfechos } \\
-26 \% \text { insatisfechos }\end{array}$ & $\begin{array}{l}\text { - } 61 \% \text { satisfechos } \\
\text { - } 30 \% \text { regularmente } \\
\text { satisfechos } \\
\text { - } 9 \% \text { insatisfechos }\end{array}$ \\
\hline Tutores & $\begin{array}{l}\text { - } 53 \% \text { regularmente } \\
\text { satisfechos } \\
\text { - } 25 \% \text { satisfechos } \\
-22 \% \text { insatisfechos }\end{array}$ & $\begin{array}{l}\text { - } 59 \% \text { regularmente } \\
\text { satisfechos } \\
\text { - } 29 \% \text { satisfechos } \\
-12 \% \text { insatisfechos }\end{array}$ & $\begin{array}{l}\text { - } 65 \% \text { regularmente } \\
\text { satisfechos } \\
-23 \% \text { satisfechos } \\
-12 \% \text { insatisfechos }\end{array}$ & $\begin{array}{l}\text { - } 68 \% \text { regularmente } \\
\text { satisfechos } \\
-19 \% \text { satisfechos } \\
-13 \% \text { insatisfechos }\end{array}$ \\
\hline Comunidad & $\begin{array}{l}-65 \% \text { satisfechos } \\
-24 \% \text { insatisfechos } \\
-11 \% \text { no responde }\end{array}$ & $\begin{array}{l}-70 \% \text { satisfechos } \\
-15 \% \text { insatisfechos } \\
-15 \% \text { no responde }\end{array}$ & $\begin{array}{l}-52 \% \text { satisfechos } \\
-33 \% \text { insatisfechos } \\
-15 \% \text { no responde }\end{array}$ & no aplica \\
\hline
\end{tabular}

Fuente: Martínez y Chavarría (2010). 
La inadecuada comunicación entre las personas encargadas de la docencia, la investigación y los usuarios, con las personas encargadas de la actualización de la biblioteca, hacen que la satisfacción de todos los usuarios, como se muestra en la Tabla N 6, en relación con la cantidad y calidad de los recursos bibliográficos disponibles sea baja.

\section{CONCLUSIONES}

La adecuada gestión administrativa de una biblioteca es un aspecto de mucha relevancia para el logro de sus objetivos y principalmente para la satisfacción de sus poblaciones meta, esta gestión influye directamente en el uso que los usuarios hagan de la misma, ya que involucra el manejo de todos los recursos necesarios para su funcionamiento entre los que se encuentran la planificación para su adecuada actualización, las instalaciones, el personal y en la actualidad un aspecto indispensable el material digital y el acceso a estos materiales.

La biblioteca es una herramienta para complementar el conocimiento de los estudiantes como para el desarrollo de la investigación. Pero para que funcione adecuadamente y cuente con el material requerido por la oferta académica de la institución y las líneas de investigación, se precisa una adecuada comunicación y vinculación con sus usuarios y proveedores.

El personal que labora en las bibliotecas debe contar con la formación suficiente, de manera que pueda prestar el servicio de manera que satisfaga a los usuarios guiándolos en el uso de la tecnología para el rápido y oportuno acceso a la información que requieren.

Las instalaciones para el desarrollo de una biblioteca en la actualidad requiere tener en cuenta aspectos como temperatura, áreas confortables tanto para el personal que labora en este lugar como para las consultas de los usuarios, e indis- pensablemente puestos con el equipamiento necesario para la consulta de material digital.

La biblioteca debe disponer del material requerido por su población meta en cuanto a su cantidad, calidad y facilidad de acceso en los medios requeridos por los usuarios, y para lograr esto se requiere el establecimiento de políticas de actualización y mejoramiento de las condiciones de estos lugares.

Las instalaciones, los equipos y el acceso a material digital deben ser suficientes para suplir las necesidades de los usuarios, y los servicios ofrecidos requieren ser apoyados por personal competente, conocedor de las últimas tecnologías y medios para acceder a la información requerida por el usuario.

Teniendo en cuenta los aspectos mencionados anteriormente y el objetivo principal de esta investigación se plantea que las bibliotecas de la UNED no están siendo eficaces en su gestión administrativa para ofrecer los servicios que requieren sus poblaciones meta.

Con el fin de mejorar se requiere establecer estrategias para conocer hasta donde están siendo efectivos los servicios ofrecidos por la biblioteca. Estas mediciones y su seguimiento se pueden lograr implementando indicadores. Estos indicadores son insumos básicos para evaluaciones que permitan detectar deficiencias que posteriormente lleven a acciones correctivas y luego de la implementación de las mismas se llegue a la mejora de los servicios bibliotecarios.

Entre los nuevos retos de las bibliotecas universitarias es importante reformular sus procesos y sus servicios para satisfacer a sus usuarios por medio del planteamiento de un cambio estratégico que permita convertir las bibliotecas tradicionales en bibliotecas híbridas de manera que sin eliminar en su totalidad sus fondos bibliográficos físicos vayan creciendo como biblioteca digital ofreciendo no solo material digital y ac- 
cesos a información por medio de internet, si no también ofreciendo servicios en línea que ayuden a los usuarios a ubicar la información que requieren, lo que implica un mayor uso de tecnologías de información en todos los procesos de la biblioteca.

Este cambio estratégico requiere la planificación del financiamiento en relación con aspectos como inversiones en equipos, espacios para su adecuada instalación y también la adquisición de bases de datos e información digital para alimentar sus fondos bibliográficos.

Adicionalmente se precisa de recursos humanos competentes no solo en el área de bibliotecología sino también en el área de las tecnologías de la información para que apoyen tanto las nuevas labores internas para la organización y clasificación de información digital como para que ofrezcan un buen servicio de apoyo, direccionamiento y formación a los usuarios.

Todos estos aspectos son importantes tenerlos en cuenta para ir hacia la mejora de los servicios bibliotecarios de acuerdo a las herramientas actuales que la tecnología esta ofreciendo pero no se puede olvidar el respeto por la propiedad intelectual y por mantener una evaluación periódica de sus servicios y productos con el fin de mejorarlos.

\section{REFERENCIAS}

ACRL Research Planning and Review Committee (2010). 2010 Top ten trends in academic libraries. College \& Research Libraries New, (71), 286-292.

Agencia para la calidad del sistema universitario de Cataluña (2002). II Plan de la Calidad de las Universidades. Guía de evaluación de bibliotecas. Cataluña: Agencia para la calidad del sistema universitario de Cataluña.

Cabral, B. (2008). La biblioteca digital y la educación a distancia como entes inseparables para incrementar la calidad de la educación. Investigación Bibliotecológica, (22) 45, 63-78.
Domínguez, M. I. (2005). La biblioteca universitaria ante el nuevo modelo de aprendizaje: docentes y bibliotecarios, aprendamos juntos porque trabajamos juntos. RED. Revista de Educación a Distancia, número monográfico II. Recuperado de http://revistas.um.es/red/ article/view/24481/23821.

Gómez, H. J. A. (2002). Tema 4 gestión de bibliotecas calidad y evaluación. Gestión de Bibliotecas, 65-66.

González, G. M. V. y Molina, P. M. (2008). Las bibliotecas universitarias: Breve aproximación a sus nuevos escenarios y retos. ACIMED, 18(2), 1-21.

Gutiérrez, C. A. y Zárate, T. F. (2007). Gestión empresarial aplicada en bibliotecas y centros de información y documentación. Ciencias de la información. Recuperado de http://pensardenuevo.org/gestión-empresarilaplicada-en-bibliotecas-y-centros-de-información- ydocumentación/

Martínez, L. y Chavarría, V (2010). Evaluación de los servicios bibliotecarios ofrecidos por la UNED para la gestión académica. CIEI Centro de Investigación y Evaluación Institucional UNED, Costa Rica.

Montagut, V. M. (2009). La biblioteca digital como apoyo a la educación a distancia. Memoria de la 3ra Conferencia Internacional de Biblioteca Digital y Educación a Distancia, 31-47.

Orera Orera, L. (2007). La biblioteca universitaria ante el nuevo modelo social y educativo. El Profesional de la Información, 16(4), 329-337. Recuperado de http:// www.elprofesionaldelainformacion.com/contenidos/2007/julio/06.pdf.

Padula, P. J. (2002). Bibliografía y bibliotecas en la educación a distancia. Biblos Revista Electrónica de Bibliotecología, Archivología y Museología, 4 (13), 1-5.

Sánchez, D. M. y Vega, V. J. (2002). Bibliotecas electrónicas, digitales y virtuales: tres entidades por definir. ACIMED, $10(6), 7$.

Tramullas, J. (2007). Bibliotecas digitales. Recuperado de http://eprints.rclis.org/11304/1/oleirosbd.pdf.

Recibido: 24 de octubre de 2012 Aceptado: 30 de octubre de 2013 